\title{
DIVISION ALGEBRAS OVER NONLOCAL HENSELIAN SURFACES
}

\author{
TIMOTHY J. FORD
}

\begin{abstract}
Let $R$ be the coordinate ring of an integral affine algebraic surface, $\widetilde{R}$ the henselization of $R$ along a reduced, connected curve and $\widetilde{K}$ the quotient field of $\widetilde{R}$. Then every central $\widetilde{K}$-division algebra $\widetilde{D}$ of exponent $n$ in $B(\widetilde{K})$ is cyclic of degree $n$. If $K$ is the quotient field of $R$ and $D$ is a central $K$-division algebra of exponent $n$ with ramification divisor $Z$ on $\operatorname{Spec} R$, then there is an étale neighborhood $U \rightarrow \operatorname{Spec} R$ of $Z$ such that upon restriction to $K(U), D$ is a cyclic algebra of exponent $n$ and index $n$.
\end{abstract}

In this paper we continue to investigate the structure of division algebras $D$ finite dimensional over their center $K$, where $K$ has transcendence degree 2 over an algebraically closed field $k$ of characteristic 0 . The motivating question behind this work, which remains unanswered, is whether the exponent of the class $[D]$ in the Brauer group $\mathrm{B}(K)$ is equal to the degree $\sqrt{(D: K)}$ of the division algebra. This question has been addressed in the works [2], [3] and [8]. In [2] it was shown that exponent $(D)=\operatorname{degree}(D)$ when exponent $(D)$ has prime factorization $2^{n} 3^{m}$. It was shown in [3] that exponent $(D)=\operatorname{degree}(D)$ when $K$ is the quotient field of the henselization at a closed point on a normal algebraic surface. Division algebras over such fields $K$ were also studied in [8]. In [8] it was shown that every central $K$-division algebra is also cyclic. That is, if exponent $(D)=n$, there is a cyclic Galois extension $L / K$ of degree $n$ which splits $D$ (see for example, $[15, \S 30]$ ). Thus a structure theory for division algebras was obtained which is similar to that of global fields. The purpose of this paper is to extend the results of [8] to the case where $K$ is the function field of a ring $\widetilde{R}$ obtained by henselizing an affine algebraic surface along a curve. The line of proof used here pretty nearly follows that of [8]. As another parallel to [8] we point out in Remark 8 that $[8, \S 2]$ can be adapted to construct the algebra $D$ as a symbol $(\alpha, \beta)_{n}$ over $K$ in the special case that $D$ ramifies on a curve $Z$ whose normalization $\bar{Z}$ is simply connected.

The results of this paper are mainly concerned with surfaces that have been henselized along a curve. For the basic properties of 
henselian couples, the reader is referred to [14]. Let $R$ be the coordinate ring of a normal, integral, 2-dimensional affine variety over $k$. Let $I$ be an ideal in $R$ such that $R / I$ is reduced and connected. Let $\widehat{R}$ be the completion of $R$ in the $I$-adic topology. Then $\widehat{R}$ is a normal domain. To see this, note first that $R$ is a G-ring [12, Theorem 77, p. 254]. Therefore $\widehat{R}$ is a normal ring [12, Theorem 79, p. 258]. Since $R / I$ is connected, it follows that $\widehat{R}$ is connected. Thus $\widehat{R}$ is a normal domain. Let $(\widetilde{R}, \widetilde{I})$ be the henselization of $R$ along $I$. By [6, Proposition 1.5], $\widetilde{R}$ is also a normal domain. We now state our main result.

THEOREM 1. Let $\widetilde{K}$ be the quotient field of either $\widehat{R}$ or $\widetilde{R}$ and $\widetilde{D}$ a central, finite dimensional $\widetilde{K}$-division algebra with $\operatorname{exponent}(\widetilde{D})=n$. Then $\widetilde{D}$ is a cyclic algebra of degree $n$.

Before starting the proof of Theorem 1 we mention an important consequence for algebras over $K$, the quotient field of $R$. For simplicity let us assume $\mathrm{B}(R)=0$ and $R$ is regular. The sequence

$$
0 \rightarrow \mathrm{B}(K) \stackrel{a}{\rightarrow} \bigoplus_{C} \mathrm{H}^{1}(\mathrm{~K}(C), \mathbb{Q} / \mathbb{Z})
$$

is exact, where the summation is over all irreducible curves $C$ on $\operatorname{Spec} R[5, \S 3]$. Therefore the class $[D]$ in $\mathrm{B}(K)$ is completely determined by the ramification data $a([D])$ in $\bigoplus \mathrm{H}^{1}(\mathrm{~K}(C), \mathbb{Q} / \mathbb{Z})$. The irreducible curves $Z_{i}$ where $a([D]) \neq 0$ make up the ramification divisor $Z=Z_{1} \cup \cdots \cup Z_{m}$ of $D$. Denote $a([D])$ by $\left(L_{1}, \ldots, L_{m}\right)$ where $L_{i}$ is a cyclic Galois extension of the function field $\mathrm{K}\left(Z_{i}\right)$ of $Z_{i}$. Again, for the sake of simplicity, assume $Z$ is connected. Suppose $I$ is a radical ideal for $Z$ and let $(\widetilde{R}, \widetilde{I})$ be the henselization of $R$ along $I$. Let $(R, I) \rightarrow(A, J)$ be an étale neighborhood of $(R, I)$. Then we can assume $A$ is a domain. Let $\mathrm{K}(A)$ be the quotient field of $A$. Let $\operatorname{Spec} A / J=W$. Then $W \cong Z$. In fact we may write $W$ as a union of irreducibles $W=W_{1} \cup \cdots \cup W_{m}$ where $W_{i} \cong Z_{i}$ for each $i$. The diagram

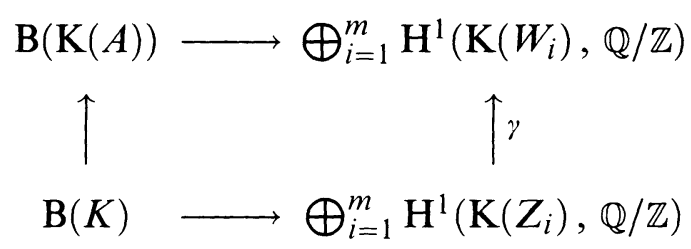

$$
\mathrm{B}(K) \longrightarrow \bigoplus_{i=1}^{m} \mathrm{H}^{1}\left(\mathrm{~K}\left(Z_{i}\right), \mathbb{Q} / \mathbb{Z}\right)
$$


commutes. Since $R \rightarrow A$ is unramified on $Z$, the vertical arrow $\gamma$ is an isomorphism. Up to the isomorphism $\gamma$, the ramification data for $D \otimes \mathrm{K}(A)$ on $\operatorname{Spec} A$ agrees with that for $D$ on $\operatorname{Spec} R$. So $D \otimes \mathrm{K}(A)$ has exponent $n$. Therefore, upon restriction to $\widetilde{K}, \widetilde{D}=$ $D \otimes \widetilde{K}$ has exponent $n$. By Theorem $1, \widetilde{D}=D \otimes \widetilde{K}$ has index $n$. More specifically, $\widetilde{D}$ is split by a cyclic extension $L / \widetilde{K}$ of degree $n$. Therefore, for some $(A, J), D \otimes \mathrm{K}(A)$ is a cyclic algebra with index $=$ exponent. This proves

COROLlARY 2. Let $R$ be the affine coordinate ring of a smooth surface with quotient field $K$ and $\mathrm{B}(R)=(0)$. Let $D$ be a central $K$ division algebra. There is an étale $R$-algebra $A$ such that upon restriction to $\mathrm{K}(A)$ the ramification data of $D$ are preserved and $D$ becomes a cyclic central simple algebra with index = exponent.

We now begin the proof of Theorem 1 . We begin with some general results about splitting the ramification of central simple algebras on surfaces. Let $S$ be a normal, integral, algebraic surface with function field $F$. Let $L$ be a finite extension field of $F$ and $Y \rightarrow S$ the integral closure of $S$ in $L$.

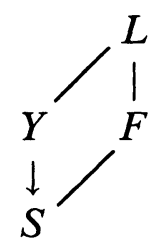

Let $\pi: Y^{\prime} \rightarrow Y$ be any desingularization of $Y$. That is, $Y^{\prime}$ is a nonsingular surface and $\pi$ is a proper, birational morphism. There is a complex

$$
\begin{aligned}
0 & \rightarrow \mathrm{B}\left(Y^{\prime}\right) \rightarrow \mathrm{B}(L) \stackrel{a}{\rightarrow} \bigoplus_{C} \mathrm{H}^{1}(\mathrm{~K}(C), \mathbb{Q} / \mathbb{Z}) \stackrel{r}{\rightarrow} \bigoplus_{P} \mu(-1) \\
& \stackrel{s}{\rightarrow} \mathrm{H}^{4}\left(Y^{\prime}, \mu\right) \rightarrow 0
\end{aligned}
$$

which is exact except possibly at the term $\bigoplus \mathrm{H}^{1}(\mathrm{~K}(C), \mathbb{Q} / \mathbb{Z})$. This follows by combining sequences (3.1) and (3.2) of [5]. If $\mathrm{H}^{3}\left(Y^{\prime}, \mu\right)=$ $0,(3)$ is exact. The first summation is over all irreducible curves $C \subseteq Y^{\prime}$, the second over all closed points $P \in Y^{\prime}$. Let $D$ be a central $F$-division algebra and $D_{L}=D \otimes L$, the restriction of $D$ to $L$. We say that $L$ splits the ramification of $D$ on $S$ if there exists a desingularization $\pi: Y^{\prime} \rightarrow Y$ such that $\left[D_{L}\right]$ is in the image of the map $\mathrm{B}\left(Y^{\prime}\right) \rightarrow \mathrm{B}(L)$. As was shown in [3], it is possible to find a 
desingularization $\sigma: S^{\prime} \rightarrow S$ such that the ramification divisor $Z$ of $D$ on $S^{\prime}$ has normal crossings. As was pointed out in $[8, \S 1]$, it is technically easier to test whether $L$ splits the ramification of $D$ on $S^{\prime}$ than on $S$. The following proposition was implicitly proved in the text immediately preceding Theorem 1.6 of [8]. We will make use of the construction used in the proof; hence we give it here for reference.

Proposition 3. With the preceding notation, if the exponent of $[D]$ in $\mathrm{B}(F)$ is $n$, then there exists a cyclic Galois extension $L / F$ of degree $n$ that splits the ramification of $D$ on $S^{\prime}$.

Proof. Let $Z$ be the ramification divisor of $D$ on $S^{\prime}$. Using [11, $\S \mathrm{V} .1]$ we can find nonsingular curves $D_{1}, D_{2}$ on $S^{\prime}$ such that $Z \sim D_{1}-D_{2}$ and the curve $Z \cup D_{1} \cup D_{2}$ is a divisor with normal crossings. So there is a function $\alpha \in F$ such that the principal divisor $(\alpha)$ has underlying curve $Z \cup D_{1} \cup D_{2}$ and $\alpha$ has valuation +1 on each irreducible component of $Z$. Let $L=F\left(\alpha^{1 / n}\right)$. Let $Y^{\prime}$ be the integral closure of $S^{\prime}$ in $L$. Let $Y^{\prime \prime} \rightarrow Y^{\prime}$ be any resolution of the singularities of $Y^{\prime}$. Since $(\alpha)$ has normal crossings $Y^{\prime}$ has only rational singularities [8, Theorem 1.2]. We want to show that the algebra $D_{L}=D \otimes L$ is unramified along each prime divisor of $Y^{\prime \prime}$, or that $D_{L}$ extends to an Azumaya algebra on $Y^{\prime \prime}$. Let $\sigma$ be the composite morphism $Y^{\prime \prime} \rightarrow Y^{\prime} \rightarrow S^{\prime}$. Then $\sigma$ has ramification index $n$ at the prime components of $Z$. If $E$ is the exceptional divisor of $Y^{\prime \prime} \rightarrow Y^{\prime}$, then $D_{L}$ is unramified on $Y^{\prime \prime}-E$ by [8, diagram (4)]. Since $Y^{\prime}$ has rational singularities $E$ is simply connected so $B\left(Y^{\prime \prime}\right) \cong B\left(Y^{\prime \prime}-E\right)$ [8, Corollary 0.2]. Thus $D_{L}$ is unramified on $Y^{\prime \prime}$ and $L$ splits the ramificiation of $D$ on $S^{\prime}$.

EXAMPLE 4. This is an example of a field extension $L / F$ that splits the ramification of $D$ but does not split the Brauer class of $D$. In the setting of Theorem 1 above, this phenomenon cannot occur because the surface $X=\operatorname{Spec} \widetilde{R}$ is henselized. Let $S=A^{2}$, the affine plane over $k, F=k(x, y)$ and $D$ the symbol algebra $(x, y)_{2}$. Let $L$ be the quadratic extension $F \sqrt{x y\left(x^{2}-1\right)\left(y^{2}-1\right)}$. The ramification divisor of $D$ on $S$ is the curve $x y=0$. Now $L$ splits the ramification of $D$ on $S$ since the ramification index of $Y^{\prime} \rightarrow S$ is 2 at the primes $(x)$ and $(y)$. So $D_{L}$ is unramified on the surface defined by the equation $z^{2}=x y\left(x^{2}-1\right)\left(y^{2}-1\right)$. We claim $D_{L}$ is not split. This is because $D_{L}$ remains unsplit upon restriction to the field $M=F\left(\sqrt{x\left(x^{2}-1\right)}, \sqrt{y\left(y^{2}-1\right)}\right)$. In fact the symbol algebra $(x, y)_{2}$ 
is a generator of ${ }_{2} B\left(C_{1} \times C_{2}\right)$ where $C_{1}$ and $C_{2}$ are the elliptic curves defined by $u^{2}=x\left(x^{2}-1\right)$ and $v^{2}=y\left(y^{2}-1\right)$ respectively (see [7, Example 9]).

As in Theorem 1 , let $R$ be the affine coordinate ring of a normal, integral, 2 dimensional variety over $k$. Let $I$ be an ideal in $R$ such that $R / I$ is reduced and connected. Let $(\widetilde{R}, \widetilde{I})$ be the henselization of $R$ along $I$. Let $\widetilde{K}$ be the quotient field of $\widetilde{R}$ and $X=\operatorname{Spec} \widetilde{R}$. Let $\widetilde{D}$ be a central $\widetilde{K}$-division algebra with exponent $n$ in $\mathrm{B}(\widetilde{K})$. Let $\pi: X^{\prime} \rightarrow X$ be a resolution of the singularities of $X$. Let $Z \subseteq X^{\prime}$ be the ramification divisor of $\widetilde{D}$ on $X^{\prime}$. If necessary, blow up points on $X^{\prime}$ so that the ramification divisor of $\widetilde{D}$ on $X^{\prime}$ is a divisor with normal crossings.

Corollary 5. Let $\pi: X^{\prime} \rightarrow X, \widetilde{K}, \widetilde{D}, n$ be as above. Then there exists a cyclic extension $L$ of $\widetilde{K}$ of degree $n$ that splits the ramification of $\widetilde{D}$ on $X^{\prime}$.

Proof. Since $\widetilde{R}$ is the direct limit of integral domains $A_{i}$ of finite type over $K$ there is an étale neighborhood $A$ of $(R, I)$ and a central simple algebra $\Lambda$ over $F=\mathrm{K}(A)$ such that $\widetilde{D}=\Lambda \otimes_{F} \widetilde{K}$. Since $U=$ $\operatorname{Spec} A$ is an algebraic surface we apply Proposition 3 to find a cyclic splitting field $E / F$ for the ramification of $\Lambda$ on $U$. Let $L=\widetilde{K} E$ and let $Y^{\prime}$ be the integral closure of $X^{\prime}$ in $L$. By the construction in the proof of Proposition 3 we see that $Y^{\prime} \rightarrow X^{\prime}$ has ramification index $n$ along each of the prime components of $Z$, where $Z$ is the ramification divisor of $\widetilde{D}$ on $X^{\prime}$. Thus $\widetilde{D}_{L}=\widetilde{D} \otimes L=\Lambda \otimes E \otimes L$ is unramified on any desingularization of $Y^{\prime}$. The construction of $E$ also makes it clear that $L / \widetilde{K}$ is cyclic of degree $n$.

Proof of Theorem 1. By approximation techniques [6] it suffices to assume $\widetilde{K}$ is the quotient field of $\widetilde{R}$. We use the notation introduced immediately before Corollary 5 . By Corollary 5 there is a cyclic extension $L$ of degree $n$ that splits the ramification of $\widetilde{D}$ on $X^{\prime}$. If $Y^{\prime}$ is the integral closure of $X^{\prime}$ in $L$ and $Y^{\prime \prime} \rightarrow Y^{\prime}$ is a resolution of the singularities of $Y^{\prime}$, then Lemma 6 below shows that $B\left(Y^{\prime \prime}\right)=0$. Thus $\widetilde{D}_{L}$ is split.

Lemma 6. Let $X=\operatorname{Spec} \widetilde{R}$ be as above. Let $\pi: X^{\prime} \rightarrow X$ be a resolution of the singularities of $X$. Then $\mathrm{B}\left(X^{\prime}\right)=\mathrm{H}^{2}\left(X^{\prime}, \mathrm{G}_{m}\right)=0$, $\mathrm{H}^{3}\left(X^{\prime}, \mathrm{G}_{m}\right)=\mathrm{H}^{3}\left(X^{\prime}, \mu\right)=0$ and $\mathrm{H}^{4}\left(X^{\prime}, \mathrm{G}_{m}\right)=\mathrm{H}^{4}\left(X^{\prime}, \mu\right)=0$. 
Before proving the lemma we state a corollary which follows immediately from (3) and Lemma 6.

COROLlaRY 7. Let $\pi: X^{\prime} \rightarrow X$ be a resolution of the singularities of $X=\operatorname{Spec} \widetilde{R}$. Let $\widetilde{K}$ be the quotient field of $\widetilde{R}$. The sequence

$$
0 \rightarrow \mathrm{B}(\widetilde{K}) \stackrel{a}{\rightarrow} \bigoplus_{C} \mathrm{H}^{1}(\mathrm{~K}(C), \mathbb{Q} / \mathbb{Z}) \stackrel{r}{\rightarrow} \bigoplus_{P} \mu(-1) \rightarrow 0
$$

is exact where the first summation is over all irreducible curves $C \subseteq X^{\prime}$ and the second over all closed points $P \in X^{\prime}$.

Proof of Lemma 6. First we note that since $X^{\prime}$ is smooth, $\mathrm{H}^{p}\left(X^{\prime}, \mathrm{G}_{m}\right)$ is torsion for $p \geq 2\left[10\right.$, p. 71]. Thus $\mathrm{H}^{p}\left(X^{\prime}, \mathrm{G}_{m}\right)=$ $\mathrm{H}^{p}\left(X^{\prime}, \mu\right)$ for $p \geq 3$ by Kummer theory. Since $X^{\prime}$ is not complete, $\mathrm{H}^{4}\left(X^{\prime}, \mu\right)=0$ [13, Cor. VI.11.5]. Since $X$ is normal it has finitely many singular points say $\xi_{1}, \ldots, \xi_{m}$. Let $g_{i}: \xi_{i} \hookrightarrow X$ be the closed immersion, for each $i$. Let $\Omega=\left\{\xi_{1}, \ldots, \xi_{m}\right\}$. Then on $X^{\prime}-\pi^{-1}(\Omega)$, $\pi$ is an isomorphism; hence the sheaves $\mathrm{R}^{q} \pi_{*}\left(\mu_{n}\right)$ have support on $\Omega$ for $q \geq 0$. By proper base change each stalk $\mathrm{R}^{q} \pi_{*}\left(\mu_{n}\right)_{\xi}$ is canonically isomorphic to $\mathrm{H}^{q}\left(X_{\xi_{1}}^{\prime}, \mu_{n}\right)$ where $X_{\xi_{1}}^{\prime}=X^{\prime} \times \xi_{i}$ is the fiber of $\pi$ over $\xi_{i}$. So $\mathrm{R}^{q} \pi_{*}\left(\mu_{n}\right)$ is the direct image sheaf $\bigoplus_{i=1}^{m} g_{i^{*}}\left(F_{i}\right)$ where $F_{i}$ is a sheaf on $\xi_{i}$ [13, Cor. II.3.11]. Since $\xi_{i}=$ Spec $k$ and $k$ is algebraically closed, $F_{i}$ is the constant sheaf $\mathrm{R}^{q} \pi_{*}\left(\mu_{n}\right)_{\xi_{i}}=\mathrm{H}^{q}\left(X_{\xi_{i}}^{\prime}, \mu_{n}\right)$. The spectral sequence for $g_{i}: \xi_{i} \hookrightarrow X$ is $\mathrm{H}^{p}\left(X, \mathrm{R}^{q} g_{i^{*}}\left(F_{i}\right)\right) \Rightarrow \mathrm{H}^{p+q}\left(\xi_{i}, F_{i}\right)$. Since $g_{i}$ is a closed immersion $\mathrm{R}^{q} g_{i^{*}}\left(F_{i}\right)=0$ for $q>0$. Thus $\mathrm{E}_{0}^{j}=\mathrm{H}^{j}\left(\xi_{i}, F_{i}\right)=\mathrm{E}_{1}^{j}=\cdots=\mathrm{E}_{j}^{j}=\mathrm{H}^{j}\left(X, g_{i^{*}} F_{i}\right)$. Again, $k$ is algebraically closed, so $\mathrm{H}^{j}\left(X, g_{i^{*}} F_{i}\right)=0$ for $j>0$. This proves Step 1.

Step 1. $\mathrm{H}^{p}\left(X, \mathrm{R}^{q} \pi_{*}\left(\mu_{n}\right)\right)=0$ for $p>0, q>0$.

Step 2. Let $Z=\operatorname{Spec} \widetilde{R} / \widetilde{I}$. Then $\operatorname{Pic} X \cong \operatorname{Pic} Z$.

This follows from [16].

Step 3. $\mathrm{B}(X)=0$ and $\mathrm{H}^{2}\left(X, \mu_{n}\right)=0$.

Since $Z$ is an affine curve, $\operatorname{Pic} Z$ is divisible. This follows from the exact Kummer sequence

$$
\operatorname{Pic} Z \stackrel{n}{\rightarrow} \operatorname{Pic} Z \rightarrow \mathrm{H}^{2}\left(Z, \mu_{n}\right)
$$

and the fact that $\mathrm{H}^{2}\left(Z, \mu_{n}\right)=0$ since $Z$ is not complete [13, Cor. VI.11.5]. By Step 2, Pic $X$ is also divisible. Now $\mathrm{B}(X) \cong \mathrm{B}(Z)=0$ [9] or [16] since $Z$ is 1 dimensional over $K$. Kummer theory gives 
the exact sequence

$$
\operatorname{Pic} X \stackrel{n}{\rightarrow} \operatorname{Pic} X \rightarrow \mathrm{H}^{2}\left(X, \mu_{n}\right) \rightarrow \mathrm{H}^{2}\left(X, \mathrm{G}_{m}\right) \stackrel{n}{\rightarrow} \cdots .
$$

But ${ }_{n} \mathrm{~B}(X)={ }_{n} \mathrm{H}^{2}\left(X, \mathrm{G}_{m}\right)$ by Gabber's theorem, so $\mathrm{H}^{2}\left(X, \mu_{n}\right)=0$. This completes Step 3.

Now let $C$ denote the fiber $X^{\prime} \times_{X} \Omega$ over the singular points of $X$. Let $C_{\text {red }}$ denote the reduced fiber and write $C_{\text {red }}=C_{1} \cup \cdots \cup C_{t}$ as a union of irreducible curves. We may assume $C_{\text {red }}$ has pure codimension one. The closed immersion $C_{\text {red }} \hookrightarrow X^{\prime}$ induces a homomorphism Pic $X^{\prime} \rightarrow \operatorname{Pic} C_{\text {red }}$. The Kummer map is Pic $C_{\text {red }} \rightarrow \mathrm{H}^{2}\left(C_{\text {red }}, \mu_{n}\right)$.

Step 4. The composite map Pic $X^{\prime} \rightarrow \operatorname{Pic} C_{\text {red }} \rightarrow \mathrm{H}^{2}\left(C_{\text {red }}, \mu_{n}\right)$ is surjective.

For each irreducible component $C_{i}$ of $C_{\text {red }}$ choose a point $P_{i}$ such that $P_{i}$ is not in the singular set of $C_{\text {red }}$. We can also assume each $P_{i}$ is not on the curve $\pi^{-1}(Z)$. Since each $C_{i}$ is nonsingular and $X^{\prime}$ is nonsingular we can find a prime divisor $V_{i}$ for each $i$ such that $V_{i}$ intersects $C_{i}$ transversally at $P_{i}$ :

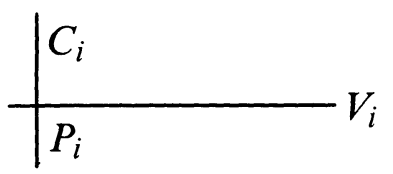

So $V_{i}$ is prime, disjoint from $\pi^{-1}(Z)$, hence is a henselian curve. Thus $V_{i}$ is geometrically unibranched and intersects $C_{\text {red }}$ exactly at $P_{i}$. Consider the diagram

$$
\begin{array}{cccccc}
1 & \mapsto & P_{i} & & \\
\mathbb{Z} & \rightarrow & \operatorname{Pic}_{i} & \rightarrow & \operatorname{Pic}\left(C_{i}-P_{i}\right) \\
\downarrow & & \downarrow & & \downarrow \\
\mathbb{Z} / n & \rightarrow & H^{2}\left(C_{i}, \mu_{n}\right) & \rightarrow & H^{2}\left(C_{i}-P_{i}, \mu_{n}\right)
\end{array}
$$

Now $\mathrm{H}^{2}\left(C_{i}-P_{i}, \mu_{n}\right)=0$ and $\mathbb{Z} / n \rightarrow \mathrm{H}^{2}\left(C_{i}, \mu_{n}\right)$ is an isomorphism. Thus (5) shows that the class of $P_{i}$ in $\operatorname{Pic} C_{i}$ maps to a generator of $\mathrm{H}^{2}\left(C_{i}, \mu_{n}\right)$. The composite $\mathrm{Pic} X^{\prime} \rightarrow \operatorname{Pic} C_{i}$ takes the class of $V_{i}$ to the class of $P_{i}$. This proves Step 4 since $\mathrm{H}^{2}\left(C_{\text {red }}, \mu_{n}\right)=$ $\bigoplus \mathrm{H}^{2}\left(C_{i}, \mu_{n}\right)$.

Step 5. $\mathrm{H}^{2}\left(X^{\prime}, \mu_{n}\right) \cong \mathrm{H}^{0}\left(X, \mathrm{R}^{2} \pi_{*}\left(\mu_{n}\right)\right)$.

Consider the spectral sequence for $\pi: X^{\prime} \rightarrow X$,

$$
\mathbf{H}^{p}\left(X, \mathbf{R}^{q} \pi_{*}\left(\mu_{n}\right)\right) \Rightarrow \mathbf{H}^{p+q}\left(X^{\prime}, \mu_{n}\right) .
$$


From Steps 1 and 3 the sequence looks like

$$
\begin{array}{lcc}
\mathrm{H}^{0}\left(X, \mathrm{R}^{2} \pi_{*} \mu_{n}\right) & 0 & 0 \\
\mathrm{H}^{0}\left(X, \mathrm{R}^{1} \pi_{*} \mu_{n}\right) & 0 & 0 \\
\mathbf{H}^{0}\left(X, \pi_{*} \mu_{n}\right) & \mathrm{H}^{1}\left(X, \pi_{*} \mu_{n}\right) & \mathbf{H}^{2}\left(X, \pi_{*} \mu_{n}\right)=0
\end{array}
$$

So $\mathrm{H}^{2}\left(X^{\prime}, \mu_{n}\right)=\mathrm{E}_{0}^{2} \supseteq \mathrm{E}_{1}^{2} \supseteq \mathrm{E}_{2}^{2}=0$. Since $\mathrm{E}_{1}^{2}=0, \mathrm{E}_{0}^{2}=\mathrm{E}_{\infty}^{0,2}=$ $\mathrm{H}^{0}\left(X, \mathrm{R}^{2} \pi_{*} \mu_{n}\right)$ and the map $\mathrm{H}^{2}\left(X^{\prime}, \mu_{n}\right) \rightarrow \mathrm{H}^{0}\left(X, \mathrm{R}^{2} \pi_{*} \mu_{n}\right)$ is an isomorphism.

Step 6. The Kummer theory map $\operatorname{Pic} X^{\prime} \rightarrow \mathrm{H}^{2}\left(X^{\prime}, \mu_{n}\right)$ is surjective.

The spectral sequence $\mathrm{H}^{p}\left(X, \mathrm{R}^{q} \pi_{*}\left(G_{m}\right)\right) \Rightarrow \mathrm{H}^{p+q}\left(X^{\prime}, \mathrm{G}_{m}\right)$ yields the exact sequence of lower degree terms

$$
0 \rightarrow \operatorname{Pic} X \rightarrow \operatorname{Pic} X^{\prime} \rightarrow \mathrm{H}^{0}\left(X, \mathrm{R}^{1} \pi_{*}\left(\mathrm{G}_{m}\right)\right) .
$$

Combining (7) with the Kummer theory maps (4) and Step 5 we get the commutative diagram

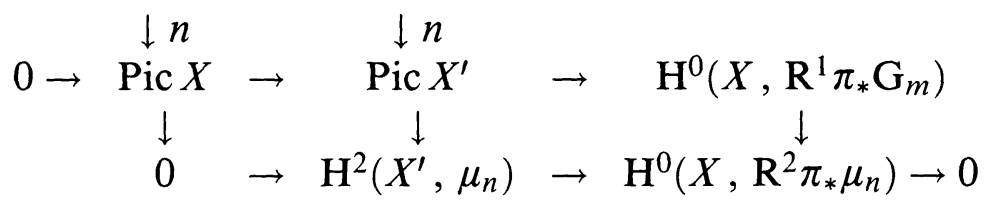

Now

$$
\begin{aligned}
\mathrm{H}^{0}\left(X, \mathrm{R}^{2} \pi_{*} \mu_{n}\right) & =\bigoplus_{i=1}^{m} \mathrm{H}^{0}\left(X, g_{i^{*}}\left(\mathrm{H}^{2}\left(X_{\xi_{t}}^{\prime}, \mu_{n}\right)\right)\right) \\
& =\bigoplus \mathrm{H}^{2}\left(X_{\xi_{1}}^{\prime}, \mu_{n}\right)=\mathrm{H}^{2}\left(C, \mu_{n}\right) .
\end{aligned}
$$

The inclusion $C_{\text {red }} \hookrightarrow C$ is defined by a sheaf of nil ideals so $\mathrm{H}^{2}\left(C, \mu_{n}\right) \rightarrow \mathrm{H}^{2}\left(C_{\mathrm{red}}, \mu_{n}\right)$ is an isomorphism [4, VIII, Cor. 1.2]. By Step 4 we see that the composite $\operatorname{Pic} X^{\prime} \rightarrow \mathrm{H}^{2}\left(X^{\prime}, \mu_{n}\right) \rightarrow$ $\mathrm{H}^{2}\left(C_{\text {red }}, \mu_{n}\right)$ is surjective. Combining the above results gives $\mathrm{Pic} X^{\prime} \rightarrow$ $\mathrm{H}^{2}\left(X^{\prime}, \mu_{n}\right)$ surjective.

Step 7. $\mathrm{B}\left(X^{\prime}\right)=\mathrm{H}^{2}\left(X^{\prime}, \mathrm{G}_{m}\right)=0$.

Since $X^{\prime}$ is a smooth surface, $\mathrm{B}\left(X^{\prime}\right)=\mathrm{H}^{2}\left(X^{\prime}, \mathrm{G}_{m}\right)$. By Kummer theory,

$$
\operatorname{Pic} X^{\prime} \stackrel{n}{\rightarrow} \operatorname{Pic} X^{\prime} \rightarrow \mathrm{H}^{2}\left(X^{\prime}, \mu_{n}\right) \rightarrow{ }_{n} \mathrm{~B}\left(X^{\prime}\right) \rightarrow 0
$$

is exact. By Step 6, $\mathrm{B}\left(X^{\prime}\right)=0$.

Step 8. $\mathrm{H}^{3}\left(X^{\prime}, \mathrm{G}_{m}\right)=\mathrm{H}^{3}\left(X^{\prime}, \mu_{n}\right)=0$.

As pointed out at the beginning of this proof, $\mathrm{H}^{3}\left(X^{\prime}, \mathrm{G}_{m}\right)=$ $\mathrm{H}^{3}\left(X^{\prime}, \mu_{n}\right)$. The spectral sequence $(6)$ yields $\mathrm{H}^{3}\left(X^{\prime}, \mu_{n}\right)=\mathrm{E}_{0}^{3}=$ $\mathrm{E}_{1}^{3}=\mathrm{E}_{2}^{3}=\mathrm{E}_{3}^{3}=\mathrm{H}^{3}\left(X, \mu_{n}\right)=0$ since $X$ is an affine surface. 
REMARK 8 . Let $(R, I),(\widetilde{R}, \widetilde{I})$ be as in Theorem 1 except assume moreover that $R$ is regular and $Z=\operatorname{Spec} R / I$ has simply connected desingularization. That is, if $\bar{Z}$ is the desingularization of $Z$, then $\mathrm{H}^{1}(\bar{Z}, \mathbb{Q} / \mathbb{Z})=0$. Let $\widetilde{K}$ be the quotient field of $\widetilde{R}$ and $\widetilde{D}$ a central $\widetilde{K}$-division algebra with exponent $n$ in $\mathrm{B}(\widetilde{K})$. Then Theorem 1 shows that $\widetilde{D}$ is cyclic, hence is a symbol algebra $(\alpha, \beta)_{n}$ over $\widetilde{K}$. Following the steps of $[8, \S 2]$ one can give an explicit description of $\alpha$ and $\beta$. The details are omitted.

REMARK 9. We close with some comments on the possibility of globalizing the above techniques to an affine rational surface with trivial Brauer group (e.g. $A^{2}$ ). In Corollary 2, suppose one can find the étale $R$-algebra $A$ such that $(\mathrm{K}(A): K)$ is prime to index $(D)$. Then, upon restriction to $\mathrm{K}(A)$ the index of $D$ remains constant by [1, p. 60]. So index $(D)=\operatorname{exponent}(D)$. To prove that such an algebra $A$ always exists does not appear to be possible in the near future. The henselian property was used in a critical way in Step 4 to lift Picard group elements from the ramification divisor. Suppose an étale neighborhood $A$ of the ramification divisor can be constructed such that (1) the degree $(\mathrm{K}(A): K)$ is prime to degree $(D)$ and (2) on $\operatorname{Spec} A$, the composite map $\operatorname{Pic}\left(\operatorname{Spec} A \times X^{\prime}\right) \rightarrow \operatorname{Pic} C_{\text {red }} \rightarrow \mathrm{H}^{2}\left(C_{\text {red }}, \mu_{n}\right)$ of Step 4 is surjective. Then, upon restriction to $\mathrm{K}(A) \quad D$ will be a cyclic algebra with index $=$ exponent. Again, this means exponent $(D)=\operatorname{index}(D)$.

\section{REFERENCES}

[1] A. A. Albert, Structure of Algebras, Amer. Math. Soc., New York, 1939, revised 1961.

[2] M. Artin, Brauer-Severi varieties, in Brauer Groups in Ring Theory and Algebraic Geometry, Lecture Notes in Math., Vol. 917, Springer-Verlag, Berlin (1982), 194-210.

[3] _ Two dimensional orders of finite representation type, preprint.

[4] M. Artin, A. Grothendieck and J. L. Verdier, SGA 4, Théorie des Topos et Cohomologie Etale des Schémas, Lecture Notes in Math., Vols. 269, 270, 305, Springer-Verlag, Berlin/New York, 1972-1973.

[5] M. Artin and D. Mumford, Some elementary examples of unirational varieties which are not rational, Proc. London Math. Soc., 25 (1972), 75-95.

[6] J. Denef and D. Harbater, Global approximation in dimension two, J. Algebra, 129 (1990), 159-193..

[7] T. Ford, On the Brauer group and the cup product map, in: Perspectives in Ring Theory (F. van Oystaeyen and Lieven Le Bruyn, Eds.), NATO ASI series, Reidel, Dordrecht, 1988.

[8] T. Ford and D. Saltman, Division algebras over henselian surfaces, Israel Mathematical Conference Proceedings 1 (1989), 320-336. 
[9] S. Greco, Algebras over nonlocal Hensel rings II, J. Algebra, 13 (1969), 48-56.

[10] A. Grothendieck, Le groupe de Brauer, I, II, III, in: Dix Exposés sur la Cohomologie des Schémas, North Holland, Amsterdam, 1968.

[11] R. Hartshorne, Algebraic Geometry, Springer-Verlag, New York, 1977.

[12] H. Matsumura, Commutative Algebra, 2nd. ed., Benjamin/Cummings, Reading, Mass., 1980.

[13] J. Milne, Etale Cohomology, Princeton University Press, Princeton, N.J., 1980.

[14] M. Raynaud, Anneaux Locaux Henséliens, Lecture Notes in Math. Vol. 169, Springer-Verlag, Berlin 1970.

[15] I. Reiner, Maximal Orders, Academic Press, New York 1975.

[16] R. Strano, On the étale cohomology of Hensel rings, Comm. Algebra, 12 (1984), 2195-2211.

Received May 8, 1989. Supported in part by the NSF under grant DMS-8620411.

Florida AtLantic University

BOCA RATON, FL 33431 